EPJ Web of Conferences 69, 00025 (2014)

DOI: $10.1051 /$ epjconf / 20146900025

(C) Owned by the authors, published by EDP Sciences, 2014

\title{
Initialization effects via the nuclear radius on transverse in-plane flow and its disappearance
}

\author{
Rajni Bansal ${ }^{1}$ and Sakshi Gautam ${ }^{2, a}$ \\ ${ }^{1}$ Department of Physics, Panjab University, Chandigarh -160 014, India. \\ ${ }^{2}$ Department of Physics, Dev Samaj College for Women, Sector 45 (B), Chandigarh -160 047, India.
}

\begin{abstract}
We study the dependence of collective transverse flow and its disappearance on initialization effects via the nuclear radius within the framework of the Isospin-dependent Quantum Molecular Dynamics (IQMD) model. We calculate the balance energy using different parametrizations of the radius available in the literature for the reaction of ${ }^{12} \mathrm{C}+{ }^{12} \mathrm{C}$ to explain its measured balance energy. A mass-dependent analysis of the balance energy through out the periodic table is also carried out by changing the default liquid drop IQMD radius.
\end{abstract}

\section{Introduction}

The collective transverse flow in heavy ion collisions is a measure of the pressure build up during the compression phase and has been used extensively to gain insight into the properties of nuclear matter at different thermodynamical conditions [1-5]. The collective transverse flow is directly connected to the dynamic evolution of the reaction, and is sensitive to the momentum dependence of the mean field [2, 3], the nucleon-nucleon cross-section [4], different equations of state (EOS) [5] and as well as to various reaction parameters such as the incident energy [6], colliding geometry [7] and mass of the colliding system $[3,7,8]$. The beam energy dependence of the collective transverse flow leads to its disappearance at a particular energy termed the balance energy [9]. The balance energy is the result of the counterbalancing of the attractive mean field (which is dominant at low incident energies) and the repulsive nucleon-nucleon scattering, which decides the fate of the reaction at higher incident energies. The balance energy (representing the vanishing of flow) is of great significance because the experimentally determined balance energy can be easily compared with various theoretical calculations as it is free from any experimental uncertainties. Detailed theoretical studies using various transport models have revealed its sensitivity to the EOS and the in-medium nucleon-nucleon crosssection as well as to various entrance channel parameters [6, 8-12]. At the same time, the collective transverse flow and its disappearance has also been found to depend on the isospin degree of freedom $[13,14]$.

As inferred from the literature, structural and initialization effects play a significant role when studying lighter systems as compared to heavier systems $[15,16]$. Thus, the initialization as well as structural effects in heavy-ion collisions at intermediate energies can be of important concern in

\footnotetext{
ae-mail: sakshigautm@gmail.com
} 
reaction dynamics. Recently, Yong et al. [16] studied initialization effects on symmetry energy sensitive observables like the free neutron to proton ratio $(\mathrm{n} / \mathrm{p})$, the $\pi^{+} / \pi^{-}$ratio and the neutron to proton differential flow, $F_{x}^{n-p}$ using different parametrizations of Skyrme forces within the framework of the Isospin-dependent Boltzmann Uehling Uhlenbeck (IBUU) model. The radius parameter plays a very crucial role in phenomena like fusion, fission, cluster radioactivity, formation of super heavy nuclei, etc. $[17,18]$. Even in the framework of the proximity potential, a suitable choice of the radius parametrization is essential to reproduce the experimental data on the fusion barrier nicely [17, 18]. Here we aim to study the role of initialization effects on the collective transverse flow and its disappearance via nuclear radii. This could also, in part, explain the experimental balance energy for the reaction of ${ }^{12} \mathrm{C}+{ }^{12} \mathrm{C}$.

\section{The model}

The IQMD model has been used extensively for studying isospin effects on a large number of observables [19]. The IQMD model is an n-body theory which simulates a heavy ion reaction on an event by event basis, hence preserves the correlations and fluctuations of the reaction. The isospin degree of freedom enters into the calculations via the symmetry potential, cross-sections, and Coulomb interaction.

In this model, baryons are represented by Gaussian-shaped density distributions given by:

$$
f_{i}(\vec{r}, \vec{p}, t)=\frac{1}{\pi^{2} \hbar^{2}} \exp \left(-\left[\vec{r}-\overrightarrow{r_{i}}(t)\right]^{2} \frac{1}{2 L}\right) \times \exp \left(-\left[\vec{p}-\vec{p}_{i}(t)\right]^{2} \frac{2 L}{\hbar^{2}}\right) .
$$

Nucleons are initialized in a sphere with radius $\mathrm{R}=1.12 \mathrm{~A}^{1 / 3} \mathrm{fm}$, in accordance with the liquiddrop model. Each nucleon occupies a volume of $h^{3}$, so that phase space is uniformly filled. The initial momenta are randomly chosen between 0 and the Fermi momentum $\left(\vec{p}_{F}\right)$. The nucleons of the target and projectile interact by two- and three-body Skyrme forces, a Yukawa potential, Coulomb interactions and momentum-dependent interactions. In addition to the use of explicit charge states of all baryons and mesons, a symmetry potential between protons and neutrons corresponding to the Bethe-Weizsacker mass formula has been included. The hadrons propagate using the Hamilton equations of motion:

$$
\frac{d \overrightarrow{r_{i}}}{d t}=\frac{d\langle H\rangle}{d \overrightarrow{p_{i}}} ; \quad \frac{d \overrightarrow{p_{i}}}{d t}=-\frac{d\langle H\rangle}{d \overrightarrow{r_{i}}}
$$

with

$$
\langle H\rangle=\langle T\rangle+\langle V\rangle \quad=\sum_{i} \frac{p_{i}^{2}}{2 m_{i}}+\sum_{i} \sum_{j>i} \int f_{i}(\vec{r}, \vec{p}, t) V^{i j}\left(\vec{r}^{\prime}, \vec{r}\right) \times f_{j}\left(\vec{r}^{\prime}, \vec{p}^{\prime}, t\right) d \vec{r} d \vec{r}^{\prime} d \vec{p} d \vec{p}^{\prime} .
$$

The baryon potential $V^{i j}$, in the above relation, reads as

$$
\begin{aligned}
V^{i j}\left(\vec{r}_{i}-\vec{r}_{j}\right)= & V_{S k y}^{i j}+V_{Y u k}^{i j}+V_{\text {Coul }}^{i j}+V_{m d i}^{i j}+V_{s y m}^{i j} \\
= & {\left[t_{1} \delta\left(\vec{r}_{i}-\vec{r}_{j}\right)+t_{2} \delta\left(\vec{r}_{i}-\vec{r}_{j}\right) \rho^{\gamma-1}\left(\frac{\vec{r}_{i}+\vec{r}_{j}}{2}\right)\right]+t_{3} \frac{\exp \left(\left|-\left(\vec{r}_{i}-\vec{r}_{j}\right)\right| / \mu\right)}{\left.\left(\mid \vec{r}_{i}-\vec{r}_{j}\right) \mid / \mu\right)}+\frac{Z_{i} Z_{j} e^{2}}{\left|\left(\vec{r}_{i}-\vec{r}_{j}\right)\right|} } \\
& +t_{4} l^{2}\left(t_{5}\left(\vec{p}_{i}-\vec{p}_{j}\right)^{2}+1\right) \delta\left(\vec{r}_{i}-\vec{r}_{j}\right)+t_{6} \frac{1}{\rho_{0}} T_{3 i} T_{3 j} \delta\left(\vec{r}_{i}-\vec{r}_{j}\right) .
\end{aligned}
$$

Here $Z_{i}$ and $Z_{j}$ denote the charges of the $i^{t h}$ and $j^{\text {th }}$ baryon, and $T_{3 i}$ and $T_{3 j}$ are their respective $T_{3}$ components (i.e., $1 / 2$ for protons and $-1 / 2$ for neutrons). The parameters $t_{1}, \ldots, t_{6}$ are adjusted 
to the real part of the nucleon optical potential. For the density dependence of the nucleon optical potential, a standard Skyrme-type parametrization is employed. We use a soft momentum-dependent (SMD) equation of state with an isospin and energy-dependent cross-section reduced by $20 \%$ i.e., $\sigma=0.8 \sigma_{\text {free }}$ and the value of $32 \mathrm{MeV}$ for the strength of the symmetry potential in the present simulations. It is worth mentioning that this choice of EOS and in-medium nucleon-nucleon crosssection is also used to reproduce the balance energy for the reactions of ${ }^{58} \mathrm{Ni}+{ }^{58} \mathrm{Ni}$ and ${ }^{58} \mathrm{Fe}+{ }^{58} \mathrm{Fe}$ for the entire collision geometry [20]. The details about the elastic and inelastic cross-sections for proton-proton and proton-neutron collisions can be found in Ref. [21]. The cross-sections for neutronneutron collisions are assumed to be equal to the proton-proton collision cross-sections. Also the neutron-proton cross-section is three times the neutron-neutron collision cross-section. Two particles collide if their minimum distance $d$ fulfills

$$
d \leq d_{0}=\sqrt{\frac{\sigma_{t o t}}{\pi}}, \sigma_{t o t}=\sigma(\sqrt{s}, \text { type }),
$$

where 'type' denotes the ingoing collision partners (N-N....). Explicit Pauli blocking is also included; i.e., Pauli blocking of the neutrons and protons is treated separately. We assume that each nucleon occupies a sphere in coordinate and momentum space. This trick yields the same Pauli blocking ratio as an exact calculation of the overlap of the Gaussians would yield. We calculate the fractions $\mathrm{P}_{1}$ and $\mathrm{P}_{2}$ of final phase space for each of the two scattering partners that are already occupied by other nucleons with the same isospin as that of the scattered ones. The collision is blocked with the probability

$$
P_{\text {block }}=1-\left[1-\min \left(P_{1}, 1\right)\right]\left[1-\min \left(P_{2}, 1\right)\right],
$$

and, correspondingly is allowed with the probability $1-\mathrm{P}_{\text {block }}$. For a nucleus in its ground state, we obtain an averaged blocking probability of $\left\langle P_{\text {block }}\right\rangle=0.96$. Whenever an attempted collision is blocked, the scattering partners maintain the original momenta prior to scattering.

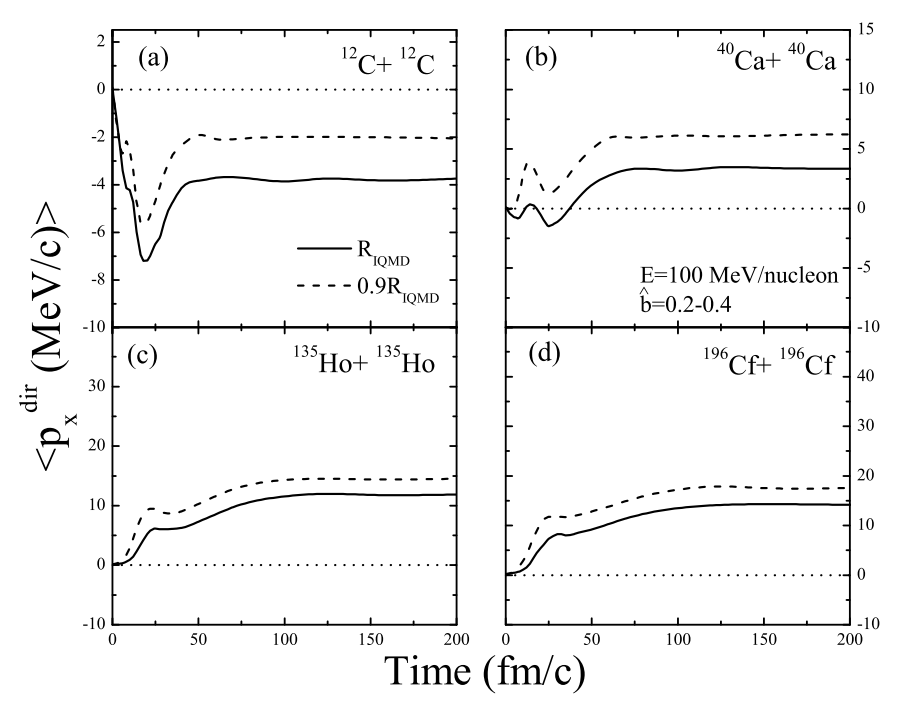

Figure 1. The time evolution of directed transverse momentum $\left(\left\langle\mathrm{p}_{x}^{\mathrm{dir}}\right\rangle\right)$ for the four different reactions at 100 $\mathrm{MeV} /$ nucleon. 


\section{Results and Discussion}

For the present study, we simulate the reactions of ${ }^{12} \mathrm{C}+{ }^{12} \mathrm{C},{ }^{24} \mathrm{Mg}+{ }^{24} \mathrm{Mg},{ }^{40} \mathrm{Ca}+{ }^{40} \mathrm{Ca},{ }^{58} \mathrm{Cu}+$ ${ }^{58} \mathrm{Cu},{ }^{96} \mathrm{Cd}+{ }^{96} \mathrm{Cd},{ }^{135} \mathrm{Ho}+{ }^{135} \mathrm{Ho}$ and ${ }^{196} \mathrm{Cf}+{ }^{196} \mathrm{Cf}$ for central collisions $\left(\mathrm{b} / \mathrm{b}_{\max }(\hat{b})=0.2-0.4\right)$ at different incident energies ranging between 40 and $140 \mathrm{MeV} /$ nucleon. A straight line interpolation is used to calculate the balance energy $\left(\mathrm{E}_{b a l}\right)$. The saturation time of the reaction varies with the mass of system.

For the transverse flow we use the quantity "directed transverse momentum $\left\langle\mathrm{p}_{x}^{\mathrm{dir}}\right\rangle$ ", which is defined as:

$$
\left\langle\mathrm{p}_{x}^{\mathrm{dir}}\right\rangle=\frac{1}{A} \sum_{i=1}^{A} \operatorname{sign}\{y(i)\} p_{x}(i),
$$

where $y(i)$ is the rapidity and $p_{x}(i)$ is the transverse momentum of the $i^{\text {th }}$ particle. The rapidity is defined as:

$$
y(i)=\frac{1}{2} \ln \frac{\mathbf{E}(i)+\mathbf{p}_{z}(i)}{\mathbf{E}(i)-\mathbf{p}_{z}(i)},
$$

where $\mathbf{E}(i)$ and $\mathbf{p}_{z}(i)$ are, respectively, the energy and longitudinal momentum of the $i^{t h}$ particle. In this definition, all rapidity bins are taken into account.

In Fig. 1, we display the time evolution of the directed transverse momentum for the reactions of ${ }^{12} \mathrm{C}+{ }^{12} \mathrm{C},{ }^{40} \mathrm{Ca}+{ }^{40} \mathrm{Ca},{ }^{135} \mathrm{Ho}+{ }^{135} \mathrm{Ho}$ and ${ }^{196} \mathrm{Cf}+{ }^{196} \mathrm{Cf}$ at an incident energy of $100 \mathrm{MeV} /$ nucleon for central collisions. The solid and dashed lines represent the calculations performed using the default liquid drop formula for the radius and reducing this radius by $10 \%$ (keeping the Fermi momentum constant), respectively. We see that the $\left\langle\mathrm{p}_{x}^{\mathrm{dir}}\right\rangle$ remains negative for the reaction of ${ }^{12} \mathrm{C}+{ }^{12} \mathrm{C}$ (Fig. 1(a)), and it remains positive for the reactions of ${ }^{135} \mathrm{Ho}+{ }^{135} \mathrm{Ho}$ (Fig. 1(c)) and ${ }^{196} \mathrm{Cf}+{ }^{196} \mathrm{Cf}$ (Fig. 1(d))for both choices of the radius. This is due to the dominance of the mean field (attractive in nature) in the reaction of ${ }^{12} \mathrm{C}+{ }^{12} \mathrm{C}$. On the other hand, nucleon-nucleon collisions dominate the reactions of ${ }^{135} \mathrm{Ho}+{ }^{135} \mathrm{Ho}$ and ${ }^{196} \mathrm{Cf}+{ }^{196} \mathrm{Cf}$, and being repulsive in nature they results in positive flow. But for the reaction of ${ }^{40} \mathrm{Ca}+{ }^{40} \mathrm{Ca}$ (Fig. 1(b)), $\left\langle\mathrm{p}_{x}^{\mathrm{dir}}\right\rangle$ is negative during the initial phase of the reaction (due to

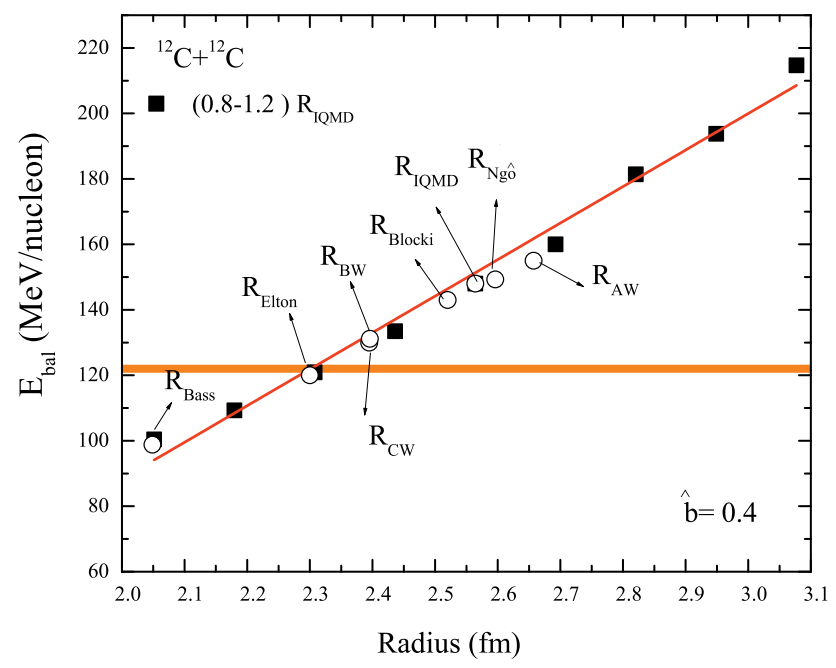

Figure 2. (Color Online) The balance energy for the reaction ${ }^{12} \mathrm{C}+{ }^{12} \mathrm{C}$ as a function of the radii of the colliding nuclei. The various symbols are explained in the text. The thick solid horizontal line represents the measured $\mathrm{E}_{b a l}$. 
the mean field), when calculated using the default radius. As the reaction proceeds, binary nucleonnucleon collisions start to take place, which in turn, increase the value of $\left\langle\mathrm{p}_{x}^{\mathrm{dir}}\right\rangle$. Also we find that the value of $\left\langle\mathrm{p}_{x}^{\mathrm{dir}}\right\rangle$ calculated using the default IQMD radius (solid line) is always smaller than the value calculated using the reduced radius (dashed line) for all four of the reactions. The increase in the flow with the decrease in radius is due to the increase in the density gradient of the nuclear matter. Also the effect of the nuclear radii is more prominent in the lighter systems $\left({ }^{12} \mathrm{C}+{ }^{12} \mathrm{C} \mathrm{\&}{ }^{40} \mathrm{Ca}+{ }^{40} \mathrm{Ca}\right)$ as it results in an approximate $70 \%$ increase in the flow, whereas in the heavier systems $\left({ }^{135} \mathrm{Ho}+{ }^{135} \mathrm{Ho} \&\right.$ ${ }^{196} \mathrm{Cf}+{ }^{196} \mathrm{Cf}$ ) the increase in the flow is only about $20 \%$ with the decrease in radius. This is because of the fact that in the lighter systems the ratio of surface diffuseness to radius is larger compared to that of the heavier systems. So, we expect this behavior of a change in the density gradient to be quite significant. Therefore, in the lighter colliding nuclei, due to the increase in the density gradient, repulsive forces (which are $\propto\left(\frac{\rho}{\rho_{0}}\right)^{\gamma}$ ) get strengthened and increase the momentum transfer in the transverse direction. Hence we can say that the radius parameter plays a very crucial role in the reaction dynamics of lighter systems.

In the past, a lot of theoretical studies have been undertaken to explain the balance energy $(122 \pm 12$ $\mathrm{MeV} /$ nucleon) for the reaction of ${ }^{12} \mathrm{C}+{ }^{12} \mathrm{C}$ measured at National Superconducting Cyclotron Laboratory (NSCL) [8]. Westfall et al. [8], for example, expressed the need of a density-dependent parametrization of the cross-section to predict the balance energy for lighter systems $\left({ }^{12} \mathrm{C}+{ }^{12} \mathrm{C}\right)$ instead of an overall reduction of the cross-section by a constant factor. In another study, Klakow et al. [10] have shown that the proper choice of the surface thickness is necessary for calculating the balance energy for the reaction of ${ }^{12} \mathrm{C}+{ }^{12} \mathrm{C}$, as a shift of $50 \mathrm{MeV} /$ nucleon is observed with a change in the surface thickness by $1 \mathrm{fm}$. The above mentioned studies were undertaken within the framework of

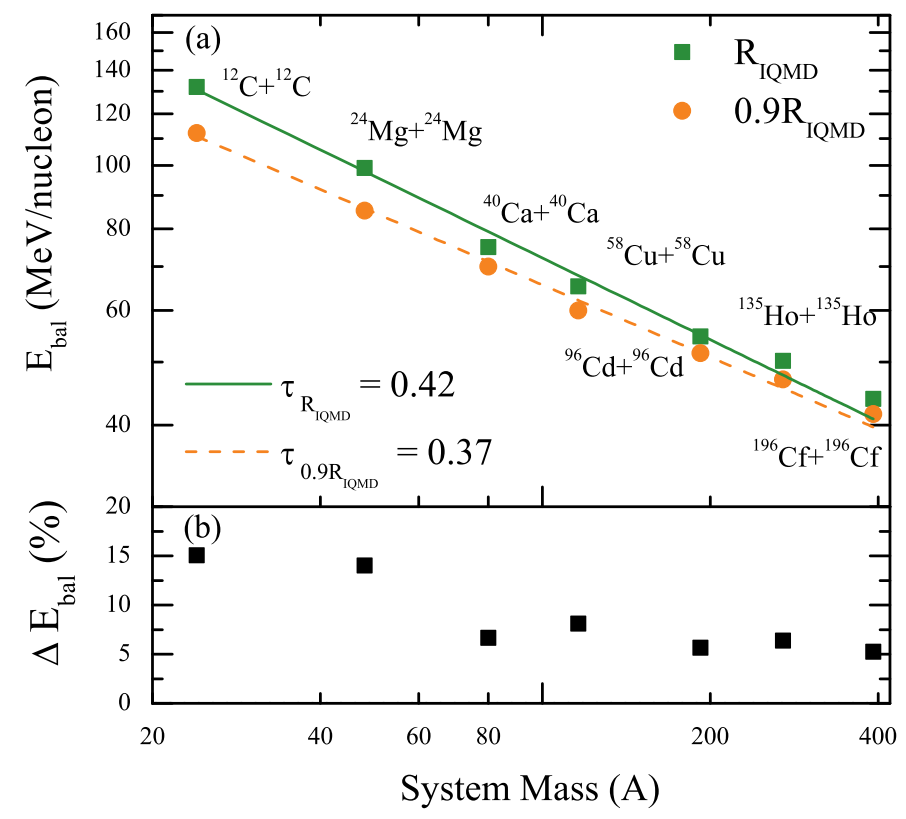

00025-p.5
Figure 3. (Color Online) The balance energy and percentage deviation as a function of the total system mass. Lines represent the power law fit $\propto \mathrm{A}^{-\tau}$. The various symbols are explained in the text. 
the Boltzmann-Uehling-Uhlenbeck (BUU) model. Puri and co-researchers have used the momentum dependence of the mean field within the Quantum Molecular Dynamics (QMD) approach to justify the power law for the entire mass range lying between ${ }^{12} \mathrm{C}+{ }^{12} \mathrm{C}$ and ${ }^{197} \mathrm{Au}+{ }^{197} \mathrm{Au}$ and stated the need of momentum-dependent interactions when dealing with lighter systems $\left({ }^{12} \mathrm{C}+{ }^{12} \mathrm{C}\right)$ as these provide necessary transverse momenta to lighter systems during the initial phase of the reaction [3]. Mota $e t$ al. [11] using a Landau-Vlasov formulation (which guarantees that good ground-state properties of finite nuclei such as binding energies and mean square radii are achieved) calculated the balance energy of ${ }^{12} \mathrm{C}+{ }^{12} \mathrm{C}$ (using free nucleon-nucleon cross-sections and a soft EOS with momentum dependence of the mean field) to be around $120 \mathrm{MeV} /$ nucleon. In the context of another study, Antisymmetrized Molecular Dynamics (AMD) model [12] calculations predicted the value of the balance energy for the reaction of ${ }^{12} \mathrm{C}+{ }^{12} \mathrm{C}$ to be around $100 \mathrm{MeV} /$ nucleon with same set of parameters used in the LandauVlasov Approach. Thus we find that different authors have given different reasons for the deviation of the balance energy of ${ }^{12} \mathrm{C}+{ }^{12} \mathrm{C}$ from the experimentally measured one and have also suggested corresponding solutions, accordingly. Since one of the initialization effects lies in the size of the nuclei (the radius of the nucleus), hence, it can also play a crucial role while extracting the balance energy for the reaction of ${ }^{12} \mathrm{C}+{ }^{12} \mathrm{C}$.

So in the next part of the paper, we also calculated the balance energy for the reaction of ${ }^{12} \mathrm{C}+{ }^{12} \mathrm{C}$ at an impact parameter of $\hat{b}=0.4$ using different parametrizations of the radius available in the literature. The choice of collision geometry is motivated by the experimentally measured balance energy [8].

In Fig. 2, we display the balance energy calculated using different parametrizations of the radius for the reaction of ${ }^{12} \mathrm{C}+{ }^{12} \mathrm{C}$. The calculated balance energies using the radius parametrization due to Bass, Brogila and Winther (BW), Christensen and Winther (CW), Blocki, IQMD, Ngô and Aage Winther (AW) are represented by open circles and also labelled respectively. We also calculated the balance energy for ${ }^{12} \mathrm{C}+{ }^{12} \mathrm{C}$ using a value of the radius $\left(2.3 \mathrm{fm}\right.$ ) for ${ }^{12} \mathrm{C}$ evaluated by Elton (labelled as Elton) [22]. The experimental datum is represented by a solid horizontal thick bar. The solid squares represent the calculated balance energy using the IQMD model radius $\left(R=r_{0} A^{1 / 3}\right)$ by varying the radius from $80 \%$ to $110 \%$ with increment of $5 \%$. The linear fit to the solid squares is shown by the solid line. We see that the balance energy increases with increasing radius. This is due to the decrease in the repulsive forces with increasing radius. Hence a larger value of the incident energy is required to counterbalance the attractive mean field. Also, the slope of the fitted line (giving the linear relationship between the balance energy and the radii of the colliding nucleus) is around $101 \pm 7$, which is large enough not to ignore any dependence of the balance energy on the radius for the ${ }^{12} \mathrm{C}+{ }^{12} \mathrm{C}$ system. We also see that the experimental datum is well reproduced when one uses the measured radius (Elton) of ${ }^{12} \mathrm{C}$ to calculate the balance energy.

Further to see the effect of the radius on the mass dependence of the balance energy, in Fig. 3(a), we display the system size dependence of the balance energy calculated using the default IQMD radius and by reducing the default radius by $10 \%$, represented by squares and circles, respectively. The lines represent the power law behavior of the mass dependence of the balance energy. We find that the balance energy decreases with a decrease in the radius due to the increase in the strength of the repulsive forces $\left(\propto \rho / \rho_{o}\right)$ with a decrease in radius. This effect is more pronounced in the lighter systems as compared to the heavier systems.

$$
\Delta \mathrm{E}_{b a l}(\%)=\left(\frac{\mathrm{E}_{b a l}^{R_{I Q M D}}-\mathrm{E}_{\text {bal }}^{0.9 R_{I Q M D}}}{\mathrm{E}_{\text {bal }}^{R_{I Q M D}}}\right) \times 100 .
$$

Fig. 3(b) displays the percentage deviation of the balance energy $\left(\Delta \mathrm{E}_{b a l}(\%)\right.$ ) (given by eq.(8)) calculated using a $10 \%$ reduced IQMD radius from the default IQMD calculations. We see that $\Delta \mathrm{E}_{b a l}(\%)$ is higher for the lighter systems when compared to heavier cases. This justifies that lighter systems 
are more sensitive to the surface effects compared to heavier ones, and thus heavier systems remain almost unaffected by the reduction of the radius.

\section{Summary}

In summary, within the framework of IQMD model using different radii for the colliding nuclei, we have demonstrated that the collective transverse flow showed a strong dependence on initialization effects for the lighter systems. Our study indicates that the radii of colliding nuclei must be treated carefully when extracting the balance energy for lighter systems.

\section{Acknowledgement}

This work has been supported by a grant from University Grants Commission (UGC), Government of India. Authors are thankful to Professor Rajeev K. Puri for enlightening discussions on the present work.

\section{References}

[1] W. Scheid, H. Muller and W. Greiner, Phys. Rev. Lett. 32, 741 (1974); H. A. Gustafsson et al., ibid. 52, 1590 (1984).

[2] J. Jaenicke et al., Nucl. Phys. A 536, 201 (1992).

[3] A. D. Sood and R. K. Puri, Eur. Phys. J. A 30, 571 (2006); S. Soff et al., Phys. Rev. C 51, 3320 (1996); R. Chugh and R. K. Puri, Phys. Rev. C 82, 014603 (2010).

[4] Y. Zhang and Z. Li, Phys. Rev. C 75, 034615 (2007); S. Kumar et al., ibid. C 58, 3494 (1998).

[5] H. Y. Zhang et al., Eur. Phys. J. A 15, 399 (2002).

[6] J. Łukasik et al., Phys. Lett. B 608, 223 (2005).

[7] A. Andronic et al., Phys. Rev. C 67, 034907 (2003).

[8] G. D. Westfall et al., Phys. Rev. Lett. 71, 1986 (1993).

[9] D. Krofcheck et al., Phys. Rev. Lett. 63, 2028 (1989).

[10] D. Kalkow, G. Welke, and W. Bauer, Phys. Rev. C 48, 1982 (1993).

[11] V. de la Mota et al., Phys. Rev. C 46, 677 (1992).

[12] A. Ono, H. Horiuchi and T. Maruyama, Phys. Rev. C 48, 2946 (1993).

[13] B. A. Li et al., Phys. Rev. Lett. 76, 4492 (1996); R. Pak et al., Phys. Rev. Lett. 78, 1022 (1997); ibid. 78, 1026 (1997); S. Gautam et al., Phys. Rev. C 82, 014604 (2010).

[14] S. Gautam and A. D. Sood, Phys. Rev. C 82, 014604 (2010); S. Gautam et al., Phys. Rev. C 83, 034606 (2011).

[15] R. Bansal, S. Gautam, R. K. Puri, and J. Aichelin, Phys. Rev. C 87, 061602(R) (2013); L. W. Chen, C. M. Ko, and B. A. Li, ibid. C 72, 064309 (2005); M. B. Tsang et al., ibid. C 86, 015803 (2012).

[16] G. C. Yong et al., Phys. Rev. C 84, 034609 (2011).

[17] I. Dutt and R. K. Puri, Phys. Rev. C 81, 064609 (2010); ibid. 064608 (2010); ibid. 047601 (2010); ibid. 044615 (2010); R. K. Gupta et al., J. Phys G: Nucl. Part. Phys. 18, 1533 (1992).

[18] I. Dutt and R. Bansal, Chin. Phys. Lett. 27, 112402 (2010).

[19] Rajni, S. Kumar, and R. K. Puri, Nucl. Phys. A 875, 173 (2012); A. Jain et al., Phys. Rev. C 85, 064608 (2012); S. Kumar et al., ibid. C 85, 024620 (2012); S. Kaur and R. K. Puri, ibid. C 87, 014620 (2013); S. Kumar et al., ibid. C 81, 014611 (2010); ibid. C 014601 (2010). 


\section{EPJ Web of Conferences}

[20] S. Gautam et al., J. Phys G: Nucl. Part. Phys. 37, 085102 (2010).

[21] C. Hartnack et al., Eur. Phys. J. A 1, 151 (1998); J. Cugnon, T. Mizutani, and J. Vandermeulen, Nucl. Phys. A 352, 505 (1981).

[22] L. R. B. Elton, Nuclear sizes (Oxford University Press, London, 1961). 